\title{
Spitzer-IRS Observations of FU Orionis Objects
}

\author{
J. D. Green ${ }^{1}$, L. Hartmann ${ }^{2}$, N. Calvet ${ }^{2}$, D. M. Watson ${ }^{1}$, M. Ibrahimov ${ }^{3}$, E. Furlan ${ }^{4}$, B. \\ Sargent $^{1}$, W. J. Forrest ${ }^{1}$ \\ 1. Department of Physics and Astronomy, University of Rochester, Rochester, NY 14627; \\ 2. Department of Astronomy, University of Michigan; 3. Ulugh Beg Astronomical Institute, \\ Uzbek Academy of Sciences, Tashkent, Uzbekistan 4. Center for Radiophysics and Space \\ Research, Cornell University, Ithaca, NY 14853-6801
}

\begin{abstract}
We present 5-35 $\mu \mathrm{m}$ spectra, taken with the Infrared Spectrograph (IRS) on the Spitzer Space Telescope, of five FU Orionis objects: FU Ori, V1515 Cyg, V1057 Cyg, BBW 76, and V346 Nor. All but V346 Nor reveal amorphous silicate grains in emission at $10 \mu \mathrm{m}$ and $20 \mu \mathrm{m}$, and show water-vapor absorption bands at 5.8 and $6.8 \mu \mathrm{m}$ and $\mathrm{SiO}$ or possibly methane absorption at $8 \mu \mathrm{m}$. These absorption features closely match these bands in model stellar photospheres signs of the gaseous photospheres of the inner regions of these objects' accretion disks. The continuum emission at $5-8 \mu \mathrm{m}$ is also consistent with such disks, and, for FU Orionis and BBW 76, longer-wavelength emission may be fit by a model which includes moderate disk flaring. V1057 Cyg and V1515 Cyg have much more emission at longer wavelengths than the others, perhaps evidence of substantial remnant of their natal, infalling envelopes.
\end{abstract}

Subject headings:

\section{Introduction}

The FU Orionis variables are pre-main sequence systems which initially were identified as having large $(\sim 5 \mathrm{mag})$, sudden outburts which typically decay in decades (e.g., Herbig 1977). FU Ori outbursts have been modeled as resulting from rapidly accreting disks in Keplerian rotation around pre-main sequence stars, a model which explains their broad infrared Spectral Energy Distributions (SEDs) and the variation of spectral type and rotational velocity with the wavelength of observation (Hartmann \& Kenyon 1996). FU Ori disks are thought to differ from those of most Classical T Tauri stars (CTTS) in that the heating of the disk is dominated by local accretion energy release (viscous dissipation), at least in the 
inner disk regions, while CTTS disks, although accreting, are thought to be heated mostly by radiation from the central star (e.g., Kenyon \& Hartmann 1987). Extended images show that FU Ori are typically surrounded by remnants of the original molecular cloud material out of which the system formed, still falling onto the disk (Herbig 1977; Goodrich 1987).

On the human time scale, FU Ori outbursts are rare events; there have only been $\sim 5-10$ outbursts detected in the past seventy years. Compared to the local rate of star formation $\sim 0.02 \mathrm{yr}^{-1}$ within $1 \mathrm{kpc}$ of the Sun (Miller \& Scalo 1979) - they happen frequently enough that it is plausible to suggest that stars commonly experience such events. Young stars may experience them repeatedly, early in their development (Herbig 1977; Hartmann \& Kenyon 1996). If so, they may play a fundamental role in early stellar protoplanetary-disk evolution: the total mass accreted in these short-lived events represents a significant fraction (10\% or more) of the final mass of the developing young star.

Mid-infrared spectra from the Infrared Spectrograph (IRS; Houck et al. 2004) on board the Spitzer Space Telescope (Werner et al. 2004) can provide crucial constraints on the structure of the inner few hundred AU of FU Ori systems. These spectra may permit isolation of the contributions of the cold envelopes and disks heated by viscous dissipation. In this paper we report IRS observations of five FU Ori objects - FU Ori, V1515 Cyg, V1057 Cyg, BBW 76, and V346 Nor - and discuss their impact on models of FU Ori objects.

\section{Observations and data reduction}

\subsection{IRS observations}

Five FU Ori objects - FU Ori, V1057 Cyg, V1515 Cyg, BBW 76, and V346 Nor - were observed between December 2003 and May 2004 using Spitzer-IRS. Table 1 is a journal of the observations. We operated the observatory in IRS Staring Mode, which includes a highaccuracy pointing peak-up using the onboard Pointing Calibration and Reference Sensor (Mainzer et al. 2003), and involves acquisition of spectra in pairs with the target displaced (nodded) along the spectrograph slit.

All of these objects were observed in both orders of the IRS Short-Low spectrograph (SL; 5.2-14 $\mu \mathrm{m}, \lambda / \Delta \lambda \sim 90$ ). BBW 76 was also observed in both orders of the IRS Long-Low spectrograph (LL; 15-40 $\mu \mathrm{m}, \lambda / \Delta \lambda \sim 90$ ). The other targets were observed in IRS ShortHigh (SH; 10-20 $\mu \mathrm{m}, \lambda / \Delta \lambda \sim 600$ ) and IRS Long-High (LH; 20-40 $\mu \mathrm{m}, \lambda / \Delta \lambda \sim 600)$. We reduced the spectra using the IRS team's Spectral Modeling, Analysis, and Reduction Tool (SMART; Higdon et al. 2004). We began with the "droop" data product from the Spitzer Science Center IRS calibration pipeline version S11.0.2, consisting of 2-D spectral-spatial, 
non-flatfielded images. The known wavelength calibration error in S11 was corrected before extraction. Before point-source spectral extraction, we corrected each pixel contained in the IRS team's mask of permanently bad or misbehaving pixels, by interpolation among its appropriately normalized neighbors. In IRS SL and LL observations both orders are always observed, but the point-source target is placed sequentially in each order, so the "off" orders provide sky-background measurements which we subtracted from the on-target spectra. We then extracted point-source spectra for each nod position using a column of variable width scaled to that of the instrumental point-spread function. For our IRS SH and LH observations we simply extracted the full slit, and did not subtract sky background emission, which is negligibly faint compared to the targets at these wavelengths.

In all cases, similarly-prepared spectra were produced from observations of two photometric standard stars, $\alpha$ Lac (A1 V) and $\xi$ Dra (K2 III). We then divided each target spectrum, nod position by nod position, by the spectrum of the one of the standard stars and multiplied by the standard's template spectrum (Cohen et al. 2003). The final spectra are averages of the nod positions, except for V346 Nor, for which only only one nod position was used. In two cases, BBW 76 and V346 Nor, slight pointing errors led to $20 \%$ flux-density discrepancies between spectra from SL and the other (wider-slit) modules, which we resolved by increasing the SL signal by a scalar factor. As no significant narrow spectral lines were detected in the high-resolution data for V1057 Cyg, FU Ori, and V346 Nor, we improved the signal-to-noise ratio in these spectra by convolution to lower spectral resolution, $\lambda / \Delta \lambda \sim 120$. We estimate the photometric accuracy of the final spectra to be approximately $5 \%$.

\subsection{Optical and near-IR photometry}

At any point in time, the continuum emission in our IRS spectra is a smooth extension of the shorter-wavelength emission. However, because FU Ori objects display short-term variation in addition to the steady decay of the outburst, comparison to shorter-wavelength observations from the same epoch as the Spitzer-IRS observations is preferred. Thus we employ UBVR observations of V1057 Cyg, FU Ori, and V1515 Cyg that were made at Maidanak Observatory throughout 2004, the continuation of a long monitoring campaign begun in 1981 for V1057 Cyg and V1515 Cyg, and in 1984 for FU Ori. Observations in this program before 2004 are available online (see Ibrahimov 1996, 1999; Clarke et al. 2005); those for 2004, contemporaneous with our Spitzer-IRS observations, are presented in tables 2, 3, and 4. The data were taken with two 60-cm Zeiss reflectors and the 48-cm AZT-14 reflector equipped with identical photon-counting photometers, reproducing the MorganJohnson system of photometry. The observations were carried out using differences with 
nearby reference stars. The RMS uncertainty of a single measurement is $\Delta V=\Delta(V-R)=$ $0.015 \mathrm{mag}, \Delta(B-V)=0.02 \mathrm{mag}$. The RMS uncertainty in U-B was approximately 0.03 mag for FU Ori, and somewhat larger for V1057 Cyg and V1515 Cyg. The resulting average visible-UV photometry for V1057 Cyg, FU Ori, V1515 Cyg and BBW 76 is displayed in table 5 .

For BBW 76, we used the visible-UV data compiled by Reipurth et al. (2002) to determine average decay rates between 1983 and 1990 for UBV photometry and linearly extrapolate values for 2004. The decay rate in the $\mathrm{B}$ and $\mathrm{V}$-band was $\sim 0.02 \mathrm{mag} \mathrm{yr}^{-1}$, while the U-band decay rate was $\sim 0.003 \mathrm{mag} y r^{-1}$. Next we calculated the decay at near-infrared wavelengths $\left(J, H\right.$, and $K_{S}$ bands) by comparison of 1983 and 1998 (2MASS) observations. This yielded a decay rate of $\sim 0.026 \mathrm{mag} y^{-1}$. We assumed this rate to apply to $R$ as well, and extrapolated to 2004 from the 1994 data in Reipurth et al. (2002).

Contemporaneous near-infrared photometry is not available for any of our targets, but comparison of older observations with those in the 2MASS point-source catalogue (PSC), as in BBW 76 above, indicate that the extrapolation from the 2MASS epoch $(\sim 1998)$ to the IRS epoch is negligibly small. Thus for V1057 Cyg, V1515 Cyg, and BBW 76 we adopt the 2MASS PSC $J, H$, and $K_{S}$ magnitudes. FU Ori is too bright to have been included in the 2MASS PSC; instead we used the 1989 near-infrared observations by Kenyon \& Hartmann (1991, henceforth KH91). Though these observations are significantly older than 2MASS, the M-band $(\lambda=4.8 \mu \mathrm{m})$ flux density matches closely at the shortest wavelengths in the IRS spectrum, indicating that FU Ori has not varied significantly at these wavelengths over the past fifteen years.

\section{Observational results}

The IRS spectra of our five program objects are displayed in Figures 1, 2, and 3. Four of the five objects - FU Ori, BBW 76, V1515 Cyg, and V1057 Cyg — have broad silicate emission features (peaking at $\lambda=10$ and $18 \mu \mathrm{m}$ ), and steeply-rising continua shortward of the silicate features. At the shorter wavelengths the spectra of these four objects are similar. In each case, the 5-9 $\mu \mathrm{m}$ continuum (Figure 2) is modulated by broad absorptions at $\lambda=$ 5.8, 6.8 and $8.0 \mu \mathrm{m}$. Based on comparisons with IRS spectra of low-mass stars (Roellig et al. 2004), and model photospheres of low-mass stars and brown dwarfs (e.g., Allard et al. 2000), we identify the two shorter wavelength features with a collection of rotation-vibration bands in gaseous $\mathrm{H}_{2} \mathrm{O}$. Calvet et al. (1991) previously noted that features between 1.4 and $2.4 \mu \mathrm{m}$ match models of water vapor absorption. Tentatively we suggest that the feature at $8 \mu \mathrm{m}$ is the fundamental rotation-vibration band of gaseous $\mathrm{SiO}$, but note that it could 
potentially be due to or influenced by absorption by methane $\left(\mathrm{CH}_{4}\right)$ as well.

Suppose that FU Ori disks exhibit gaseous features at effective temperatures $>1400 \mathrm{~K}$, but only featureless dust continuum at lower effective temperatures. The continuum corresponds to the hottest dust temperatures seen in CTTS disks (Muzerolle et al. 2003). In this case the dust opacity dominates the gaseous opacity at temperatures $\lesssim 1400 \mathrm{~K}$. From the standard steady, blackbody FU Ori disk model with a large outer radius (Kenyon et al. 1988), we estimate that almost half of the flux at $6 \mu \mathrm{m}$, and about a third of the flux at $8 \mu \mathrm{m}$, is contributed by annuli hotter than $1400 \mathrm{~K}$. This model predicts that we should detect gaseous features at these wavelengths in FU Ori objects, diluted by dust continuum emission from cooler disk regions. The appearance of strong emission due to dust at just slightly longer wavelengths (Figure 1) warns us that the 5-9 $\mu \mathrm{m}$ continuum emitted by dust grains in cooler parts of the accretion disk is probably still significant here, as previously found in the spectra of CTTSs (Furlan et al. 2005). However, neither suitable models nor observed spectra of disk photospheres at mid-infrared wavelengths yet exist for assessment of the dust-continuum dilution of the gaseous features. The spectra of FU Ori, BBW 76, V1515 Cyg, and V1057 Cyg are not as similar at the longer IRS wavelengths: the Cygnus objects are quite a bit redder at $\lambda=15-40 \mu \mathrm{m}$ than the other two, indicating a greater predominance in these objects of emission by colder dust.

\subsection{V346 Normae}

In contrast to the others, V346 Nor shows strong silicate absorption, consistent with its large visual extinction; Graham \& Frogel (1985) estimate $A_{V} \sim 6.2$ mag. Figure 3 is a comparison of IRS spectra for V346 Nor and a typical Class I young stellar object, IRAS 04016+2610 (Watson et al. 2004). The spectra are quite similar, and thus the identity of the features is the same as in Class I objects, namely water ice $(\lambda=6.0 \mu \mathrm{m})$, carbon dioxide ice $(\lambda=15.2 \mu \mathrm{m})$, a feature usually associated with methanol ice $(\lambda=6.8 \mu \mathrm{m})$, and amorphous silicates $(\lambda=10,18 \mu \mathrm{m})$.

\subsection{V1057 Cygni}

The $10 \mu \mathrm{m}$ silicate feature of V1057 Cyg appears different from the others. As we show later, after correction by the adopted $A_{V}=3.7$, using a reddening curve from Savage \& Mathis (1979) the difference vanishes (Figure 4). Thus the appearance of the feature is due to selective extinction, rather than difference in dust composition. After subtracting 
a continuum derived from the 6-8 $\mu \mathrm{m}$ region, and deriving an emissivity based on a single temperature model for the dust grains (Sargent et al. 2006), we find that the peak of the silicate emission is at $9.5 \mu \mathrm{m}$, which is indicative of amorphous silicates of pyroxene composition. This is consistent with dust composition common in CTTS disks (Demyk et al. 2000; Sargent et al. 2006), and within the usual range of composition of interstellar dust. We have not attempted such a fit to the silicate features of the other objects in the study; their silicate features have approximately the same peak wavelength and width as those of V1057 Cyg (see Figure 4). They all appear smooth and should yield composition similar to V1057 Cyg.

\subsection{V1515 Cygni}

As is common in the Cygnus clouds, the line of sight toward V1515 Cyg intersects a great deal of foreground and background nebulosity, and as a result the spectrum exhibits many spectral features from ions, molecules and small dust grains. Most prominent are the infrared bands identified with polycyclic aromatic hydrocarbons (PAHs), at $\lambda=6.2,7.7,8.6$ and $11.3 \mu \mathrm{m}$. This emission is removed almost completely by the off-order sky subtraction carried out for SL spectra during data reduction, and thus does not appear in the spectrum shown in Figure 1. That it subtracts away so precisely is an indication that the emitting material is neither associated with, nor excited by, V1515 Cyg. The same is true for [Ne II] emission at $\lambda=12.8 \mu \mathrm{m}$. For wavelengths longward of $14 \mu \mathrm{m}$, observed only with SH and LH, we lack a nearby blank-sky spectrum for subtraction, and thus spectral lines from extended nebulosity appear in Figure 1: [S III] $(18.7 \mu \mathrm{m}$ and $33.4 \mu \mathrm{m}), H_{2}(28.2 \mu \mathrm{m})$, and [Si II] $(34.8 \mu \mathrm{m})$. We expect that all these lines therein would subtract away as precisely as [Ne II], the SH profile of which we leave in Figure 1 for comparison.

\section{Discussion}

\subsection{Emission by the hot inner disk: comparison to simple models}

To compare the SEDs of the four moderately-reddened FU Ori objects with disk models, it is necessary to make extinction corrections, as most of the disk luminosity is radiated at visible wavelengths. Extinction corrections are uncertain because these systems do not have single, well-defined effective temperatures; this leads to larger uncertainties in SED shapes and system luminosities. We apply extinction corrections to the ground-based photometry (Savage \& Mathis 1979) and the IRS spectra, assuming that the optical depth at $9.7 \mu \mathrm{m}$ 
$\tau_{9.7}=A_{V} / 18$ (Schutte et al. 1998; Draine 2003). These extinction values are reasonably consistent with previous estimates in the literature but may differ by as much as 0.2 to 0.5 magnitudes in $A_{V}$. This in turn leads to an uncertainty in the peak of the SED, generally around $1 \mu \mathrm{m}$ or a little less, of order $20-30 \%$, with a similar uncertainty in the system luminosity for a given distance. The resulting, corrected spectra appear in Figure 4.

In Figure 4, we also show SEDs for steady accretion disk models assuming no inclination along the line-of-sight. The most common steady accretion disk models (e.g., Hartmann 1998) assume the disk radiates as a blackbody at a temperature that varies with radius $\mathrm{R}$ according to

$$
T_{d}^{4}=\frac{3 G M \dot{M}}{8 \pi R^{3} \sigma}\left[1-\left(\frac{R_{i}}{R}\right)^{1 / 2}\right]
$$

The maximum temperature of the disk is then given by

$$
T_{\max }=0.488\left(\frac{3 G M \dot{M}}{8 \pi R_{i}^{3} \sigma}\right)^{1 / 4}
$$

which occurs at

$$
R_{\max }=\frac{49}{36} R_{i}
$$

Thus the total luminosity can be calculated:

$$
L_{\nu}=\int_{R_{\text {in }}}^{R_{\text {out }}} \pi S_{\nu} T_{d}(R) 2 \pi R d R
$$

assuming no inclination along the line-of-sight. Rather than using blackbodies $\left(S_{\nu}=B_{\nu}(T)\right)$ we used photospheric emission computed using the photometric colors of standard stars taken from Table A5 of Kenyon \& Hartmann (1995) to provide a slightly more realistic estimate of real disk flux density at short wavelengths (blackbodies are used at long wavelengths and for all wavelengths at disk temperatures below $3000 \mathrm{~K}$ ). The same disk model is used for FU Ori, BBW 76, and V1515 Cyg, slightly scaled in luminosity, for ease of comparison; for V1057 Cyg we have used a disk model with a slightly lower $T_{\max }$ (table 6). Steady disk models are in fair to good agreement with the observations shortward of about $6 \mu \mathrm{m}$, but at longer wavelengths there is more flux at longer wavelengths than would be predicted by of the models.

Disks heated from the outside will have higher temperatures in surface layers than deeper in, a property which produces silicate emission features (Calvet et al. 1992). In contrast, 
FU Ori objects have optical and near-infrared absorption features which are interpreted as resulting from internal heating by viscous dissipation (Hartmann \& Kenyon 1985, 1987a,b). All the FU Ori objects in Figure 4 show silicate emission features at 10 and $18 \mu \mathrm{m}$, whereas at shorter wavelengths absorption bands are observed. It therefore seems likely that the spectrum longward of about $8 \mu \mathrm{m}$ or so should be considered in terms of irradiation, either of a flared disk or an envelope. We therefore first analyze the short-wavelength IRS SEDs in terms of accretion disk models and then consider irradiation of disks and envelopes for explaining the longer wavelength region in section 4.3 below.

\subsection{Disk models with radially-variable accretion rate}

FU Ori stands out in Figure 4 because its SED appears to be a bit narrower, with less infrared emission, than a standard steady disk model. This had been found before (e.g., KH91, Figure 5) without explicit comment. Of course, as FU Ori objects vary, they cannot be represented precisely by steady accretion disks. Moreover, thermal instability models of FU Ori outbursts, which can explain rapid rise times (Bell \& Lin 1994; Bell et al. 1995), predict that only an inner region of the disk participates in the outburst; this implies that, beyond a certain radius, the accretion rate is lower, reducing the amount of long-wavelength emission from outer, cooler regions. Thus we have also constructed simple disk models of this type, with results as shown in Figure 5.

The upper left panel of Figure 5 shows the FU Ori SED with various disk models. Since the Bell \& Lin (1994) and the Bell et al. (1995) FU Ori models predict a much higher accretion rate in outburst within $\sim 20 R_{\odot}$ than outside this radius (where the accretion rates tends toward the supplied infall rate), a first approximation might be to assume that the outer disk accretion rate is so much lower that the disk is effectively truncated at some radius. However, even within the limitations of the data, it is clear that disk models with substantial accretion rates must extend well past this radius to explain the observations. Steady disk models need to extend out to $\sim 100 R_{\odot}$ or more to explain our FU Ori spectra. A more realistic approximation in the thermal instability models would be to assume a lower but non-zero accretion rate, steady disk temperature distribution outside of the radius at which the thermal instability is triggered. As shown in the upper left panel of Figure 5, a model in which the accretion rate is arbitrarily dropped by a factor of ten at $\sim 30 R_{\odot}$ does a reasonably good job of joining to the IRS spectrum. Again, this requires an accretion rate in the disk which is two orders of magnitude higher than typical T Tauri rates $\left(\sim 10^{-8} \mathrm{M}_{\odot} \mathrm{yr}^{-1}\right.$ in Taurus; Calvet et al. 2004), out to $100 R_{\odot}$ or more.

These models of FU Ori have higher maximum temperatures, $7710 \mathrm{~K}$, than the 7200 
K adopted by Kenyon et al. (1988). Part of the difference may be due to the adoption by Kenyon et al. (1988) of a constant inner temperature interior to $1.36 R_{*}$, whereas our steady accretion disk models have the peak temperature at this radius and then decline inwards assuming a slowly-rotating central star; (see Hartmann 1998). We have also examined the effect of dropping the maximum temperature to correspond to a decrease in extinction of $\Delta A_{V}=0.3$ in visual magnitudes (certainly within the uncertainties), also shown in Figure 5. Although the new model disks are less luminous, with a lower $T_{\max }$ of $7140 \mathrm{~K}$, there is little difference in the model comparison at long wavelengths, because the SED shifts slightly toward longer wavelengths, compensating for the decrease in system luminosity. Thus the conclusion that the disk of FU Ori must be rapidly accreting out to of order $100 R_{\odot}$ or more appears to be insensitive to plausible changes in the extinction estimate.

Also shown in Figure 5 is a comparison of the SED of BBW 76 with a steady disk model similar to that used for FU Ori - that is, a disk model with the same maximum temperature, but with a slightly smaller inner radius (Table 6). The optical photometry does not match the disk model as well as in FU Ori. On the other hand, the extended steady disk model which passes through the recent 2MASS data points joins fairly well to the IRS spectrum. Again, the conclusion is that BBW 76 has relatively high accretion rates out to radii of order $100 R_{\odot}$ or more.

As shown in Figure 4, the steady disk model which passes through the 2MASS photometric points in V1515 Cyg extrapolates very well to the short-wavelength end of the IRS spectrum. This may be somewhat misleading, as there is evidence for a contribution from the dust disk or envelope in the $6-8 \mu \mathrm{m}$ region in addition to the contribution from the steady accretion disk (see following subsection). The IRS spectrum of V1057 Cyg clearly falls well above the extrapolation of a steady disk model, but this excess is probably due to the envelope or disk regions responsible for the far-infrared excess and silicate emission (see next subsection).

\subsection{Long-wavelength dust emission and envelopes}

Adams et al. (1987) suggested that FU Ori might have a tenuous spheroidal envelope, based in part on IRAS observations. KH91 combined IRAS data with other IR observations to study the longer-wavelength regions in FU Ori objects, with special emphasis on FU Ori, V1057 Cyg, and V1515 Cyg, and found that the infrared spectrum of FU Ori might be fit by a disk without an envelope. KH91 concluded that V1057 Cyg and V1515 Cyg needed to have an additional dusty envelope subtending a significant solid angle, which contributed strongly at wavelengths $\gtrsim 10 \mu \mathrm{m}$. KH91 suggested that this dusty structure represented infalling 
material which replaced the disk mass accreted in each outburst as a way of obtaining multiple outbursts in the lifetime of each FU Ori object. Further investigations of model envelopes were carried out by Turner et al. (1997). The IRS data provide an opportunity to reexamine more precisely the question of disk vs. envelope irradiation.

It may not be obvious that an envelope need be invoked, as T Tauri disks can have SEDs that flatten out beyond $\lambda \gtrsim 10 \mu \mathrm{m}$ (e.g., Kenyon \& Hartmann 1987; Chiang \& Goldreich 1997; D'Alessio et al. 1999). However, in the case of T Tauri disks, the irradiating source is the star, not the inner disk, and this makes a crucial difference. Consider the geometry shown in Figure 6, where for simplicity we have indicated a wedge of material which captures all the radiation emitted at angles $\theta>\theta_{m}$. In a more realistic disk/envelope combination or a flared disk, this absorbed radiation would be distributed over a wider range of radii, such that the height of the disk or the envelope at $R$ is $H$. Assuming an isotropically-emitting central source, the fraction of the central luminosity $f_{*}$ absorbed within $R, H$ is simply the fractional solid angle subtended by the envelope or disk region as seen from the central source (e.g., Hartmann 1998),

$$
f_{*}=\int_{\theta_{m}}^{\pi / 2} d \theta \sin \theta=\cos \theta_{m},
$$

where $\theta_{m}=\arctan (R / H)$. On the other hand, if the radiating source is a flat disk, even if the emergent intensity is assumed to be isotropic, the emitted flux is peaked in the direction perpendicular to the disk, due to the projection of the emitting area in the line of sight. For a geometrically flat disk with luminosity $L_{d}$, the apparent luminosity at a viewing angle $\theta$ is

$$
L(a p p)=2 L_{d} \cos \theta
$$

Thus, the fraction of the radiation from a flat inner disk, much smaller in radius than $R$, that can be intercepted by the structure in Figure 6 is

$$
f_{d}=\int_{\theta_{m}}^{\pi / 2} d \theta 2 \cos \theta \sin \theta=\cos ^{2} \theta_{m} .
$$

A typical, strongly-flared T Tauri disk might have an aspect ratio of about $H / R=0.2$ and thus could intercept about $20 \%$ of the light from its central star (equation 5 ). On the other hand, the same disk around the flat inner irradiating disk can intercept only about $4 \%$ of the inner disk luminosity (equation 7 ). Thus, large long-wavelength excesses due to irradiation by a flat disk require dusty structures which subtend much larger solid angles than would be the case if the central object radiated isotropically.

Thus, unlike a star, the apparent luminosity of a flat disk can be as much as a factor of two larger than its true luminosity if seen pole-on (equation 6). In particular, V1515 Cyg and 
V1057 Cyg have much smaller values of projected rotational velocity, $v \sin i$, than those of $\mathrm{FU}$ Ori or BBW 76 (Hartmann \& Kenyon 1985, 1987a,b; Reipurth et al. 2002), which suggests that the former two objects are observed much closer to pole-on. This suggestion is reinforced by the analysis of Goodrich (1987), who argued for similar low viewing inclinations for V1057 Cyg and V1515 Cyg on the basis of outflow envelope morphology. To the extent that the long-wavelength excess is produced in an envelope which emits more isotropically than the inner disk, the envelope will appear to absorb a smaller fraction of the total luminosity in a pole-on system than in reality.

We now consider the case of V1057 Cyg. The disk model shown in Figure 4 corresponds to a total flux received at the Earth of $\sim 1.5 \times 10^{-8} \mathrm{erg} \mathrm{cm}^{-2} \mathrm{~s}^{-1}$. The total observed flux in the IRS range is about $3.6 \times 10^{-9} \mathrm{erg} \mathrm{cm}^{-2} \mathrm{~s}^{-1}$. If we assume that all of the flux at $5 \mu \mathrm{m}$ is due to the accretion disk and not to the dusty structure responsible for the long-wavelength excess, and extrapolate this accretion disk flux as $\nu F_{\nu} \propto \nu^{4 / 3}$ to longer wavelengths or lower frequencies, then the excess above this disk emission is about $2 \times 10^{-9}$ $\mathrm{erg} \mathrm{cm}^{-2} \mathrm{~s}^{-1}$. However, as discussed above, there is evidence for dust excesses contributing at short wavelengths in V1057 Cyg; in addition, the IRS SED shows no signs of turning down at the longest wavelengths, so we have underestimated the total long-wavelength emission. We adopt $\sim 3 \times 10^{-9} \mathrm{erg} \mathrm{cm}^{-2} \mathrm{~s}^{-1}$ as an estimate of the total long-wavelength excess; this is $20 \%$ of the apparent disk luminosity. Using equation (7),

$\cos \left(\theta_{m}\right) \sim(0.2)^{1 / 2}$ or $\theta_{m} \sim 63^{\circ}$, so $H / R \sim \tan \left(90-\theta_{m}\right) \sim 0.5$. This is considerably larger aspect ratio than for most known $\mathrm{T}$ Tauri disks. In addition, the transition in the height of the absorbing dust structure must be fairly abrupt to explain the SED. We conclude, as did KH91, that there is an additional dusty envelope to explain the far-infrared excess. The true aspect ratio could be even larger if we are viewing V1057 Cyg nearly pole-on and the envelope radiates more isotropically than the inner disk.

At the other extreme, consider FU Ori. Assuming that the accretion disk emission dominates at $5 \mu \mathrm{m}$, which seems reasonable, and extrapolating this steady disk spectrum as before, the long-wavelength excess is approximately $2 \%$ of the total system luminosity. Even if we assume that all of the flux in the IRS range arises from an excess component, this constitutes no more than $4 \%$ of the total luminosity. According to equation (7), this excess in principle can be explained by absorption in a modestly flared disk with $H / R \lesssim 0.2$. We therefore reinforce the conclusion of KH91 that the SED of FU Ori can be explained by a flared disk without significant envelope contributions in the IRS wavelength range.

V1515 Cyg has a SED which is quite similar in shape to that of V1057 Cyg (Figure 4 ), but with a bit smaller long-wavelength excess. The excess over the extrapolation of the steady disk model is about $10 \%$, and the total flux in the IRS range is about $14 \%$ of 
the total luminosity; again, the SED shows no sign of turning down at long wavelengths so there must be significant longer-wavelength flux that we are not including. Adopting an estimated excess of about $12 \%$ implies $H / R \sim 0.37$, larger than typical of $\mathrm{T}$ Tauri disks. In addition, V1515 Cyg has an extremely low $v \sin i$, implying a very low angle of viewing. As discussed above, this suggests that we are underestimating the relative magnitude of the long-wavelength excess by overestimating the central luminosity, by perhaps as much as a factor of two; this would increase the required $H / R$ even more, similar to that of V1057 Cyg. We therefore conclude that V1515 Cyg must also have a dusty envelope which dominates the long-wavelength infrared emission.

BBW 76 represents an ambiguous case. The shape of its SED is more similar to that of FU Ori than to V1057 Cyg (Figure 4), but with a considerably larger excess. The excess above the steady disk model is about $5 \%$ of the total luminosity; this is very sensitive to the disk model assumed, as the the total emission in the IRS range is about $10 \%$ of the total. If we conservatively estimate a total excess of $6 \%$, then $H / R \sim 0.25$. Thus it is not clear whether some additional, perhaps relatively optically-thin envelope needs to be added to a flared disk to explain the IRS SED.

Bell \& Lin (1994), Bell et al. (1995), and Bell et al. (1997) constructed detailed models for FU Ori objects based on specific mechanisms for the outburst and parameters. They pointed out that the inner disk might exhibit departures from purely flat geometry. However, it seems unlikely that this will have a major effect on the above analysis; any regions which have "walls" which are more face-on to the envelope or disk are not likely to be large in extent, and we have neglected limb-darkening, which will reduce the flux at large $\theta$ even more than we have assumed. Bell et al. also constructed a number of disk and envelope models to explore the long-wavelength SEDs of FU Ori objects; however, these models have many parameters and it is not entirely clear how to apply their results to our new observations. The above analysis has the virtue of being minimally model-dependent while illustrating the basic geometry.

Our suggestion of envelopes that intercept significant amounts of radiation from the central disk in V1057 Cyg and V1515 Cyg is supported by recent observations in the K-band with the Keck interferometer (Millan-Gabet et al. 2006), who found that these objects are significantly more extended than predicted by the accretion disk model. Millan-Gabet et al. estimated that their observations could be explained if $3-12 \%$ of the K-band flux in V1057 Cyg and 5 - 14\% of the K flux in V1515 Cyg arises from an extended source that is well-resolved (i.e., bigger than 1 AU or so), as the KH91 envelope model would predict. The approximate amount of K-band excess, on the order of $5-10 \%$ of the central source $\mathrm{K}$ luminosity, is in reasonable agreement with the intercepted fractions of disk luminosity on 
the order of $10 \%$ derived above, assuming a moderately large $(\sim 0.3-0.5)$ effective albedo at K. In contrast, FU Ori, which has much less far-infrared excess, shows little sign of being extended at $\mathrm{K}$ in interferometric measurements, and matches the predictions of accretion disk models quite well (Malbet et al. 2005).

In estimating the amount of solid angle that the envelope must span from the observed long-wavelength emission, we have included only that emission in the IRS range, i.e. for wavelengths $\leq 35 \mu \mathrm{m}$. Given that the long-wavelength SEDs of V1057 Cyg and V1515 Cyg are nearly flat, this is clearly an underestimate of the true total excess emission. (We do not use the IRAS fluxes because they very likely include extended, non-envelope emission due to the large beam sizes.) If the SEDs were to remain flat out to $\sim 100 \mu \mathrm{m}$, as in many Class I sources, the total envelope excesses and thus the inferred solid angle subtended by the envelope could be significantly larger. Longer-wavelength observations, perhaps with MIPS or SOFIA, could help considerably in the characterization of the geometry of the circumstellar dust in these objects.

\subsection{Envelope model}

To explore the possible parameters of remnant envelopes, we explore an approximate calculation and compare it with the observations of V1057 Cyg. The models shown in Figure 7 combine a simple steady accretion disk assuming blackbody radiation, plus a flattened envelope with a polar hole in the density distribution, similar to that used in Osorio et al. (2003). The flattened density of the envelope is consistent with gravitational collapse of a sheet beginning in hydrostatic equilibrium as discussed in Hartmann et al. (1994) and Hartmann et al. (1996), referred to as $\eta$ models. The model parameters include $\eta=R_{\text {outer }} / H$, the ratio of the envelope's outer radius to the scale height (Hartmann et al. 1996); $\rho_{1}$, the density of the infalling envelope at $1 \mathrm{AU}$ given a spherical non-rotating envelope; $R_{c}$, the centrifugal radius, the innermost radius on the plane of the disk on which infalling material can be deposited. In addition, we include a polar hole cut out of the density distribution whose boundary is given by

$$
z=a\left(r_{2}-r_{1}\right)
$$

where $z$ is the height above the equatorial plane and $r_{2}$ and $r_{1}$ are radial distances; $r_{1}$ signifies the position where the envelope edge intersects the disk. The system is then viewed at inclination $i$. A schematic of this model is displayed in Figure 8.

In order to calculate the temperature structure of the envelope, we assume radiative equilibrium, using a single central source. We calculate the temperature of each spherical

shell using a density averaged over all angles (Kenyon et al. 1993). The luminosity of the 
central source is given by the steady accretion models in table 6 . The dust destruction radius is taken to be at $\sim 1400 \mathrm{~K}$, corresponding to the sublimation temperature of silicates in a low-density environment (D'Alessio 1996). The specific intensity at each wavelength is calculated by solving the radiative transfer equation along rays that thread the envelope, using the angle-dependent density distribution, as in Kenyon et al. (1993) and Hartmann et al. (1996). The source function is determined with a mean intensity from the spherical case.

Use of the spherical average radiative equilibrium to determine the temperature distribution is no longer self-consistent when a (bipolar) hole is present. We adjust the luminosity of the central source in the spherical calculation so that the total luminosity of the envelope integrated over all solid angle is approximately that expected for the particular envelope geometry heated by a flat disk, as discussed in the previous section.

The dust in the envelope uses the standard grain size distribution of the ISM: $n(a) \alpha$ $a^{-3.5}$. The grain radii are between $a_{\min }=0.005 \mu \mathrm{m}$ and $a_{\max }=0.3 \mu \mathrm{m}$. We use a mixture of dust grains from Pollack et al. (1994), as well as a mixture from Draine \& Lee (1984) known as "astronomical silicates." The grains are assumed to be spherical and we calculate their absorption efficiency factor $Q_{a b s}$ using Mie scattering code (Wiscombe 1979). The optical properties of the compounds are taken from Warren (1984), Begemann et al. (1994), and Pollack et al. (1994).

In Figure 7 we compare the results of two model envelope calculations to the observations of V1057 Cyg, for $i=45^{\circ}$. The short dashed curve indicates the contribution of the steady blackbody accretion disk. This model does a reasonably good job for the long-wavelength IRS range but has too little flux shortward of $10 \mu \mathrm{m}$. The long-dashed curve is for a model with the same parameters except for $r_{1}=3 \mathrm{AU}$ and $R_{c}=10 \mathrm{AU}$; this model is too bright in most of the IRS range though it matches the short-wavelength end better. We suspect that if we had included the heating of the disk by the envelope (e.g., D'Alessio et al. 1997), the 5 $-8 \mu \mathrm{m}$ region of the first model would be in much better agreement with the observations.

Given the complexity of the situation, the envelope parameters derived for the envelope are not definitive but merely suggestive that envelope models can account for the long-wavelength IRS fluxes in V1057 Cyg and V1515 Cyg, as suggested by KH91. Further progress on this question will require full two-dimensional axisymmetric radiative transfer calculations. 


\subsection{Envelopes and outbursts}

The envelopes we have suggested for V1057 Cyg and V1515 Cyg apparently cover modest solid angles as seen from the central sources. This implies that the (bipolar) outflow holes driven by the winds of these objects have a wide opening angle. Theories of magnetocentrifugally-driven winds from young stellar objects suggest that such winds might have a significant opening angle but have a strong dependence of ram pressure on distance from the rotation axis, such that the jets frequently seen in such objects constitute not the entire flow but merely the densest and most energetic part of a broader flow (e.g., Shu et al. 1995; Matzner \& McKee 1999).

During much of the protostellar phase, the accretion rates are small; since the mass loss rate tends to track the accretion rate (e.g., Calvet 1998), the outflow is relatively weak in this phase. An FU Ori outburst occurs when the mass accretion rate increases, perhaps by as much as three to four orders of magnitude; in consequence, lower-density, wider-angle portions of the wind can now drive out the infalling envelope. In this picture, it is no surprise that the FU Ori objects have wide-angle envelope holes. V1057 Cyg and V1515 Cyg may be relatively younger, or have had fewer outbursts, than FU Ori and BBW 76, and thus retain envelope material at smaller radii/larger covering solid angles.

Of course, each FU Ori outburst is limited in time so that only a small fraction of a protostellar envelope would be driven off by any given event. The time evolution of the envelope geometry in a situation with repeated outbursts could be quite complicated. In particular, some protostellar sources clearly have narrow outflow holes, which may indicate they have not experienced outbursts, or have particularly large envelope infall rates and thus higher infall ram pressures. The implications of this picture require further exploration.

If infall and outflow were spherical, ram pressure balance would occur for the condition

$$
\dot{M}_{\text {wind }} v_{\text {wind }}=\dot{M}_{\text {infall }} v_{\text {infall }} \text {. }
$$

Consider, for example, V1057 Cyg. KH91 assumed an envelope infall rate of $\sim 4 \times 10^{-6}$ $\mathrm{M}_{\odot} \mathrm{yr}^{-1}$ in their envelope model for $\mathrm{V} 1057 \mathrm{Cyg}$, with an inner radius of about $7 \mathrm{AU}$. At that radius, the infall velocity is (for a $0.5 M_{\odot}$ central object) $\sim 8 \mathrm{~km} \mathrm{~s}^{-1}$. With a typical wind velocity of order $300 \mathrm{~km} \mathrm{~s}^{-1}$ or more (Croswell et al. 1987), a mass loss rate of $\dot{\mathrm{M}} \gtrsim 10^{-7}$ $\mathrm{M}_{\odot} \mathrm{yr}^{-1}$ would suffice to blow out the envelope. As the mass loss rates during peak accretion rates should be closer to $10^{-5}-10^{-6} \mathrm{M}_{\odot} \mathrm{yr}^{-1}$, it is clear that even a relatively small off-axis wind component will suffice to drive out envelope material. 


\section{Conclusions}

We have presented IRS spectra of five of the best-known FU Ori objects. One of these sources, V346 Nor, shows ice absorption features and a large mid-IR excess, indicating that it is embedded within a cold icy envelope consistent with its very infrared colors. The other four program objects are moderately-reddened and show many features in common. In these objects, the IRS spectra provide direct evidence of a gaseous accretion disk through the presence of absorption features, while silicate emission features indicate the presence of outer, cooler dust.

A dusty flared disk probably can explain the long-wavelength dust emission of two of the objects (FU Ori and BBW 76); this suggests that FU Ori events can ignite even as the protostellar envelope is dispersing. Flared disk models have difficulties with accounting for the large excesses longward of 10 microns in V1057 Cyg and V1515 Cyg; instead, we conclude that an outer dusty envelope is necessary to explain the observations, as originally suggested by KH91, and which can provide the extended structure in scattered light detected in near-infrared interferometry by Millan-Gabet et al. (2006). One important feature of the envelope model is that it requires an outflow hole with a large opening angle; we suggest that such a wide outflow-driven cavity is a result of the high mass loss rate accompanying rapid accretion in the FU Ori outburst state. Further near-infrared interferometry with better imaging properties could confirm our picture of the envelopes around V1057 Cyg and V1515 Cyg. This would help support the overall picture of FU Ori outbursts occurring during the late phases of protostellar collapse.

The authors thank Alice Quillen and Judy Pipher for many helpful conversations during the data reduction and analysis. This work is based on observations made with the Spitzer Space Telescope, which is operated by the Jet Propulsion Laboratory, under NASA contract 1407. Support for this work was provided by NASA through contract 1257184 issued by JPL/Caltech and through the Spitzer Fellowship Program, under award 011 808-001. This research was supported in part by Jet Propulsion Laboratory (JPL) contract 960803 to Cornell University, and Cornell subcontracts 31419-5714 to the University of Rochester. The research of LH and NC is supported in part by NASA grant NAG5-13210. In this work

we make use of data products from the Two Micron All Sky Survey, which is a joint project of the University of Massachusetts and the Infrared Processing and Analysis Center/California Institute of Technology, funded by the National Aeronautics and Space Administration and the National Science Foundation. This research has also made use of the SIMBAD database, operated at CDS, Strasbourg, France. 


\section{REFERENCES}

Adams, F. C., Lada, C. J., \& Shu, F. H. 1987, ApJ, 312, 788

Allard, F., Hauschildt, P. H., \& Schweitzer, A. 2000, ApJ, 539, 366

Begemann, B., Dorschner, J., Henning, T., Mutschke, H., \& Thamm, E. 1994, ApJ, 423, L71

Bell, K. R., \& Lin, D. N. C. 1994, ApJ, 427, 987

Bell, K. R., Lin, D. N. C., Hartmann, L. W., \& Kenyon, S. J. 1995, ApJ, 444, 376

Bell, K. R., Cassen, P. M., Klahr, H. H., \& Henning, T. 1997, ApJ, 486, 372

Calvet, N. 1998, American Institute of Physics Conference Series, 431, 495

Calvet, N., Hartmann, L., \& Kenyon, S. J. 1991, ApJ, 383, 752

Calvet, N., Magris, G. C., Patino, A., \& D’Alessio, P. 1992, Revista Mexicana de Astronomia y Astrofisica, 24, 27

Calvet, N., Muzerolle, J., Briceño, C., Hernández, J., Hartmann, L., Saucedo, J. L., \& Gordon, K. D. 2004, AJ, 128, 1294

Chiang, E. I., \& Goldreich, P. 1997, ApJ, 490, 368

Clarke, C., Lodato, G., Melnikov, S. Y., \& Ibrahimov, M. A. 2005, MNRAS, 361, 942

Cohen, M., Megeath, S. T., Hammersley, P. L., Martín-Luis, F., \& Stauffer, J. 2003, AJ, 125,2645

Croswell, K., Hartmann, L., \& Avrett, E. H. 1987, ApJ, 312, 227

D’Alessio, P. 1996, Ph.D. Thesis

D’Alessio, P., Calvet, N., \& Hartmann, L. 1997, ApJ, 474, 397

D’Alessio, P., Calvet, N., Hartmann, L., Lizano, S., \& Cantó, J. 1999, ApJ, 527, 893

Demyk, K., Dartois, E., Wiesemeyer, H., Jones, A., D'Hendecourt, L., Jourdain de Muizon, M., \& Heras, A. M. 2000, ESA SP-456: ISO Beyond the Peaks: The 2nd ISO Workshop on Analytical Spectroscopy, 183

Draine, B. T. 2003, ARA\&A, 41, 241 
Draine, B. T., \& Lee, H. M. 1984, ApJ, 285, 89

Furlan, E., et al. 2005, ApJ, 628, L65

Goodrich, R. W. 1987, PASP, 99, 116

Graham, J. A., \& Frogel, J. A. 1985, ApJ, 289, 331

Hartmann, L. 1998, Accretion processes in star formation / Lee Hartmann. Cambridge, UK ; New York : Cambridge University Press, 1998. (Cambridge astrophysics series ; 32) ISBN 0521435072.,

Hartmann, L., \& Kenyon, S. J. 1985, ApJ, 299, 462

Hartmann, L., \& Kenyon, S. J. 1987, ApJ, 312, 243

Hartmann, L., \& Kenyon, S. J. 1987, ApJ, 322, 393

Hartmann, L., \& Kenyon, S. J. 1996, ARA\&A, 34, 207

Hartmann, L., Boss, A., Calvet, N., \& Whitney, B. 1994, ApJ, 430, L49

Hartmann, L., Calvet, N., \& Boss, A. 1996, ApJ, 464, 387

Herbig, G. H. 1977, ApJ, 217, 693

Higdon, S. J. U., et al. 2004, PASP, 116, 975

Houck, J. R., et al. 2004, ApJS, 154, 18

Ibrahimov, M. A. 1996, Informational Bulletin on Variable Stars, 4285, 1

Ibrahimov, M. A. 1999, Informational Bulletin on Variable Stars, 4691, 1

Kenyon, S. J., \& Hartmann, L. 1987, ApJ, 323, 714

Kenyon, S. J., \& Hartmann, L. W. 1991, ApJ, 383, 664

Kenyon, S. J., \& Hartmann, L. 1995, ApJS, 101, 117

Kenyon, S. J., Hartmann, L., \& Hewett, R. 1988, ApJ, 325, 231

Kenyon, S. J., Calvet, N., \& Hartmann, L. 1993, ApJ, 414, 676

Mainzer, A. K. Young, E. T., Huff, L. W., \& Swanson, D. 2003, SPIE, 4850, 122.

Malbet, F., et al. 2005, A\&A, 437, 627 
Matzner, C. D., \& McKee, C. F. 1999, ApJ, 526, L109

Millan-Gabet, R., et al. 2006, ApJ, 641, 547

Miller, G. E., \& Scalo, J. M. 1979, ApJS, 41, 513

Muzerolle, J., Calvet, N., Hartmann, L., \& D’Alessio, P. 2003, ApJ, 597, L149

Osorio, M., D’Alessio, P., Muzerolle, J., Calvet, N., \& Hartmann, L. 2003, ApJ, 586, 1148

Pollack, J. B., Hollenbach, D., Beckwith, S., Simonelli, D. P., Roush, T., \& Fong, W. 1994, ApJ, 421, 615

Reipurth, B., Hartmann, L., Kenyon, S. J., Smette, A., \& Bouchet, P. 2002, AJ, 124, 2194

Roellig, T. L., et al. 2004, ApJS, 154, 418

Sargent et al., ApJ, in press

Savage, B. D., \& Mathis, J. S. 1979, ARA\&A, 17, 73

Schutte, W. A., et al. 1998, A\&A, 337, 261

Shu, F. H., Najita, J., Ostriker, E. C., \& Shang, H. 1995, ApJ, 455, L155

Turner, N. J. J., Bodenheimer, P., \& Bell, K. R. 1997, ApJ, 480, 754

Warren, S. G. 1984, Appl. Opt., 23, 1206

Watson, D. M., et al. 2004, ApJS, 154, 391

Werner, M. W., et al. 2004, ApJS, 154, 1

Wiscombe, W. J. 1979, Mie Scattering Calculations: Advances in Technique and Fast, Vector-Speed Computer Codes, NCAR Tech. Note 140+STR (Boulder: NCAR) 

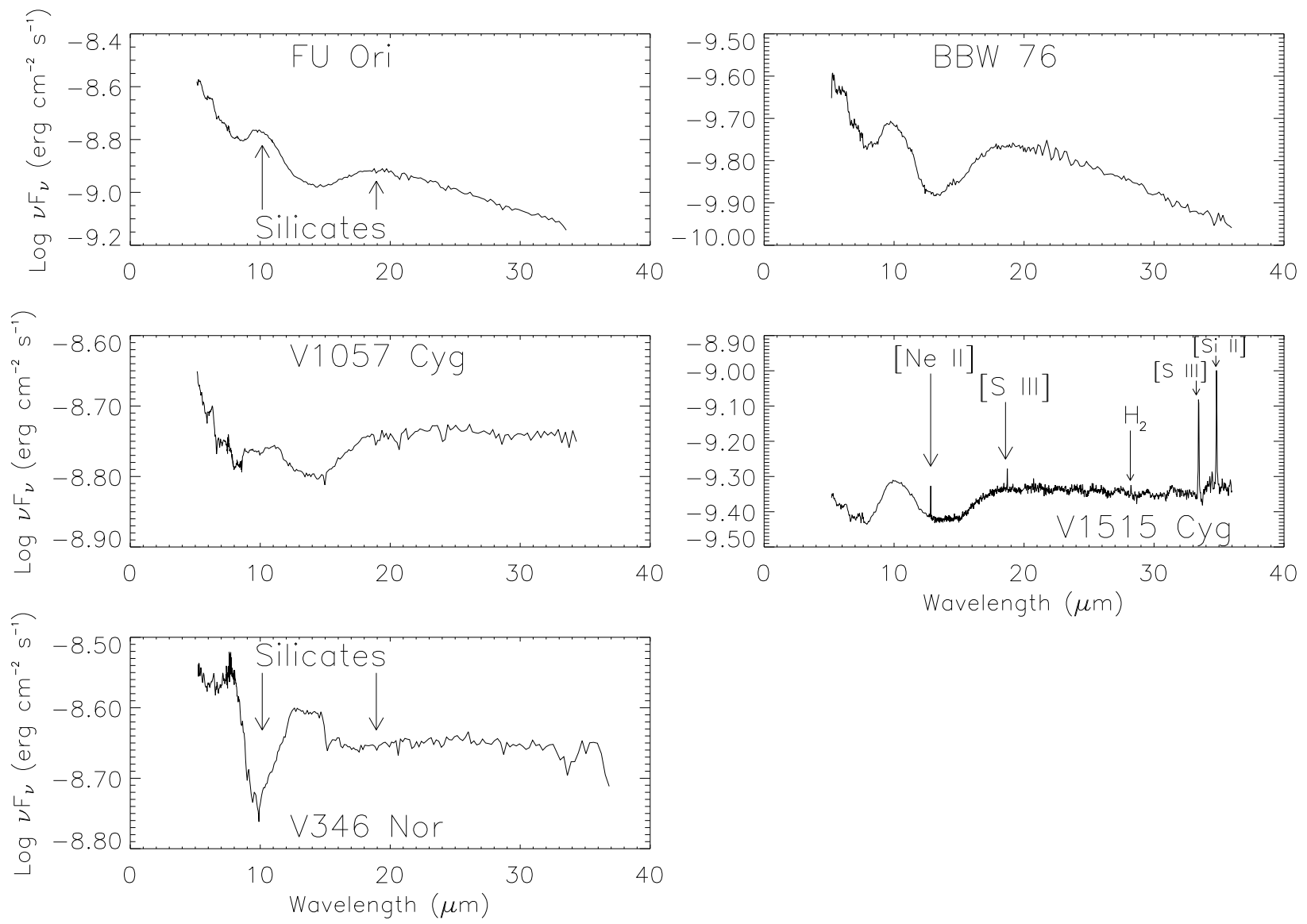

Fig. 1.- Observed IRS spectra of FU Orionis variables, uncorrected for reddening. The data for BBW 76 and V1515 Cygni are shown at full resolution: BBW 76 was a low resolution observation, and we show the high resolution data for V1515 Cygni in order to display the spectral lines. The data for the other three objects have been rebinned to low resolution. As discussed in the text, the ionic emission lines in V1515 Cyg arise in the foreground or the background. 


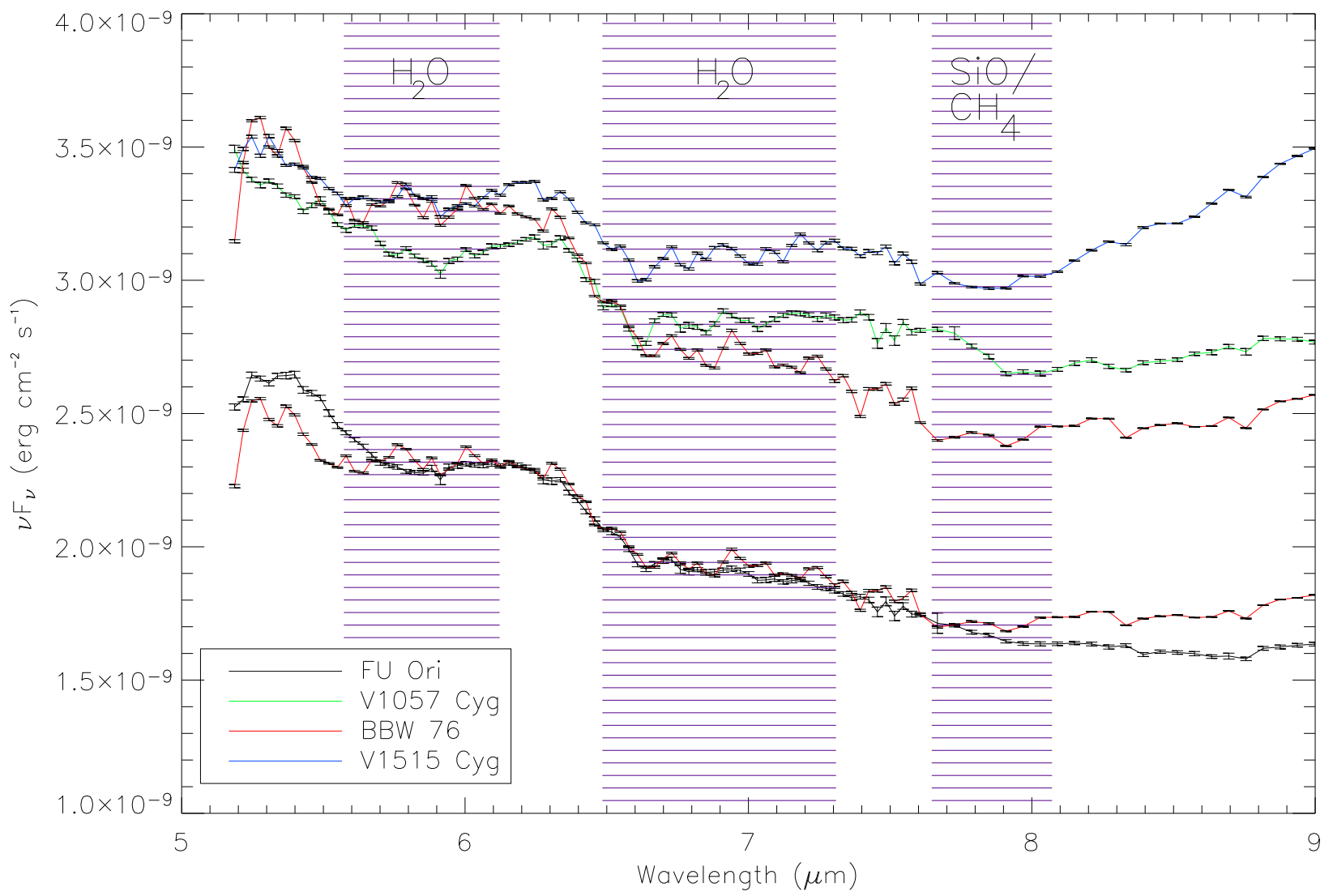

Fig. 2.- Comparison of the $5-8 \mu \mathrm{m}$ region of the four FU Ori objects showing gaseous absorption features: $\mathrm{H}_{2} \mathrm{O}$ absorption bands 5.8 and $6.8 \mu \mathrm{m}$ and an $\mathrm{SiO}$ band at $8 \mu \mathrm{m}$. The spectra have been scaled, and BBW 76 is shown twice at different scales for comparison to the sources with more emission at longer wavelengths (V1057 Cyg, V1515 Cyg), and to the source it most closely resembles (FU Ori). The error bars in the plot are derived from the difference of the two nod positions. 


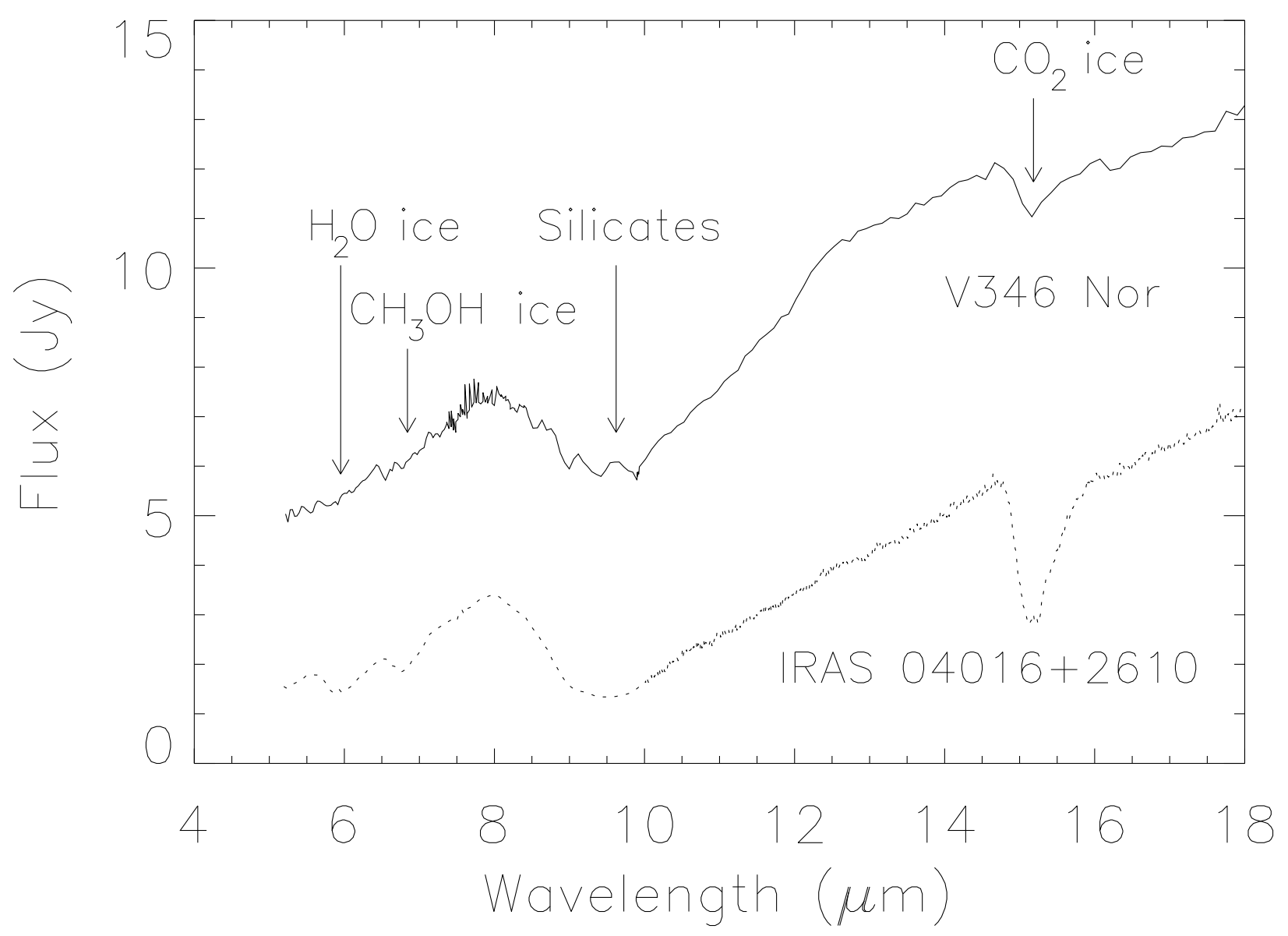

Fig. 3.- Comparison of the 5-18 $\mu \mathrm{m}$ region of V346 Nor to the Class I YSO IRAS 04016+2610 (Watson et al. 2004). The spectra are unscaled, although V346 Nor has been rebinned to low resolution. The spectrum of V346 Nor is considerably more noisy despite being brighter, because of significant telescope pointing errors. 

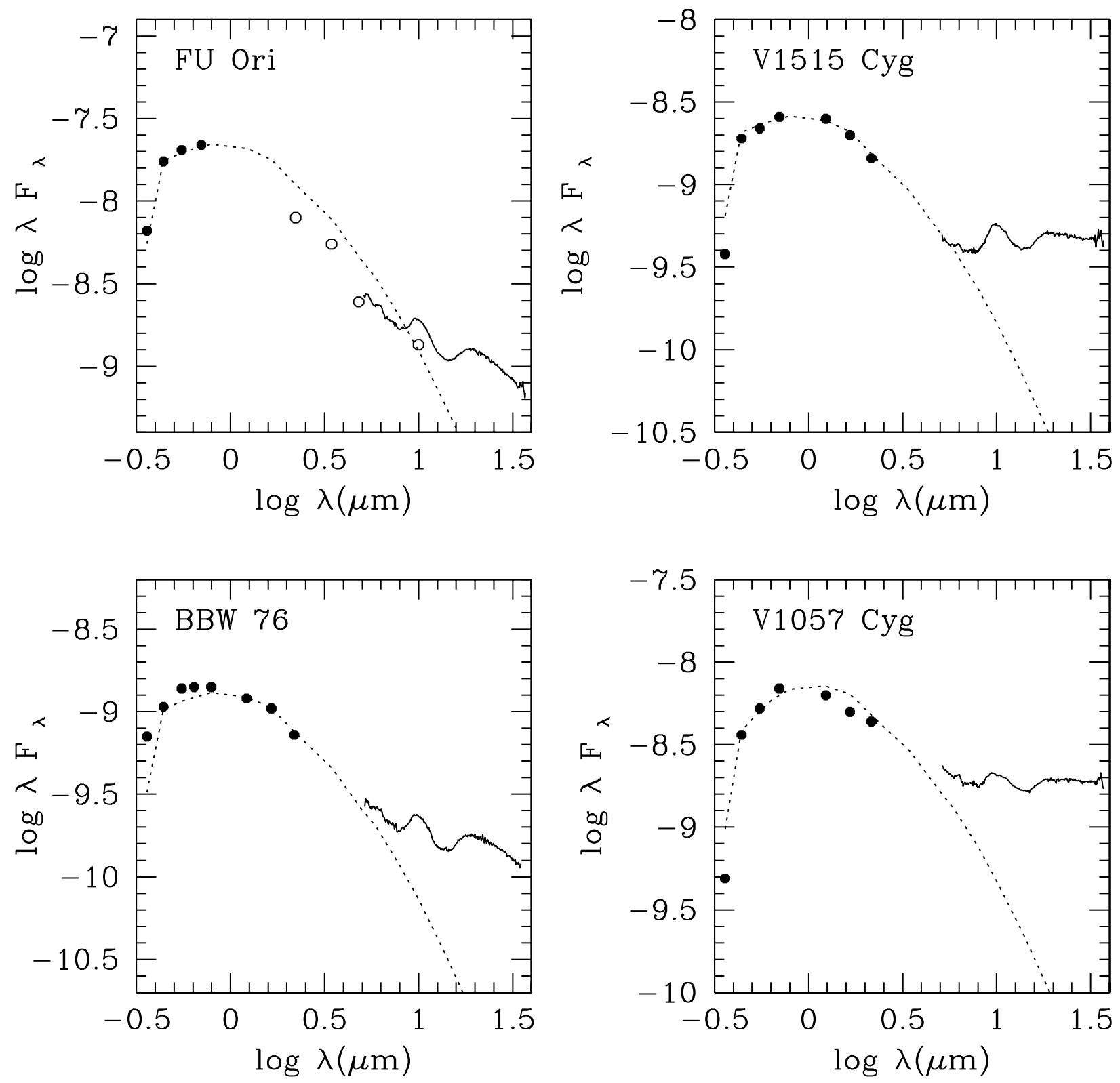

Fig. 4.- Dereddened IRS spectra (solid line) and older optical and near-IR ground-based data (circles); the dashed line is a fit from the steady accretion model using standard photometric colors down to $3000 \mathrm{~K}$ from Kenyon \& Hartmann 1995 (Table A5) with fit parameters listed in Table 6. Below $3000 \mathrm{~K}$, blackbodies are used. The value of $A_{V}$ for each object is listed in Table 5. The filled optical data circles are observations made at Maidanak Observatory during 2004; the filled near-IR data circles are 2MASS observations circa 1998; the open circles are 1989 KLMN bolometer data from KH91. 

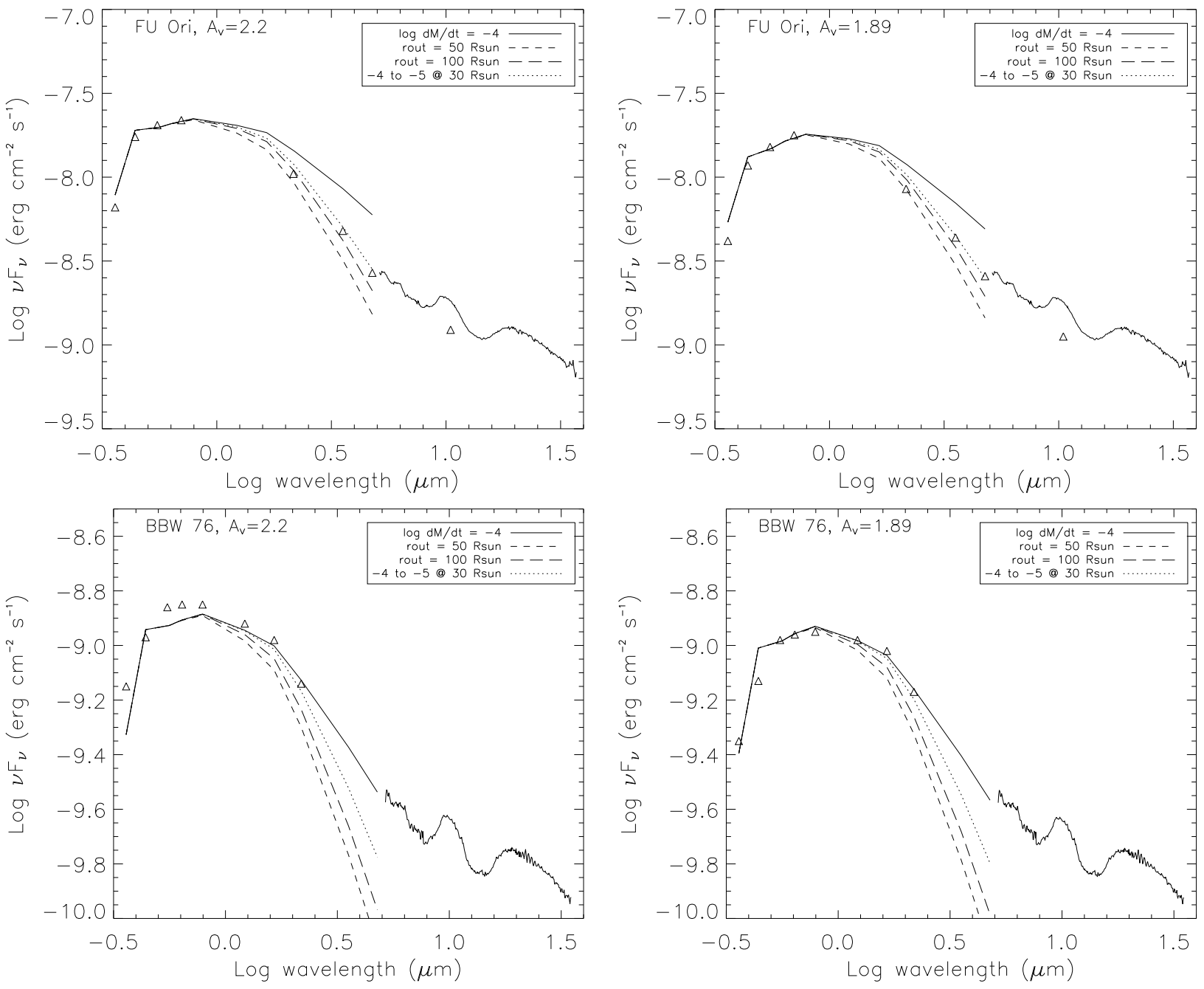

Fig. 5.- Comparison of different disk models of FU Orionis with varying conditions in the outer disk. The solid line represents a disk with a constant accretion rate of $10^{-4} M_{\odot} y r^{-1}$. The dotted line represents a model where the accretion rate is decreased to $10^{-5} M_{\odot} y r^{-1}$ beyond $30 R_{\odot}$. The longer dashed line represents a disk which is truncated beyond 100 $R_{\odot}$, and the disk represented by the shorter dashed line is truncated beyond $50 R_{\odot}$. Upper left: The model shown for FU Ori uses the values given in table 6. Upper right: The same comparison after applying a lower extinction correction, assuming $A_{V}=1.89$, and reducing $M \dot{M}$ from 1.5 to 1.1 . For this model, $T_{\max }=7140 \mathrm{~K}$. Lower left: The same comparison for BBW 76. The parameters are taken from table 6 . Lower right: $M \dot{M}$ has been reduced from 0.72 to 0.65 , consequently reducing $T_{\max }$ to $7140 \mathrm{~K}$. The optical photometry in the case of BBW 76 does not match the disk model as well as it did for FU Ori, but the extended steady disk model which passes through the 2MASS JHK data connects well to the IRS spectrum. 


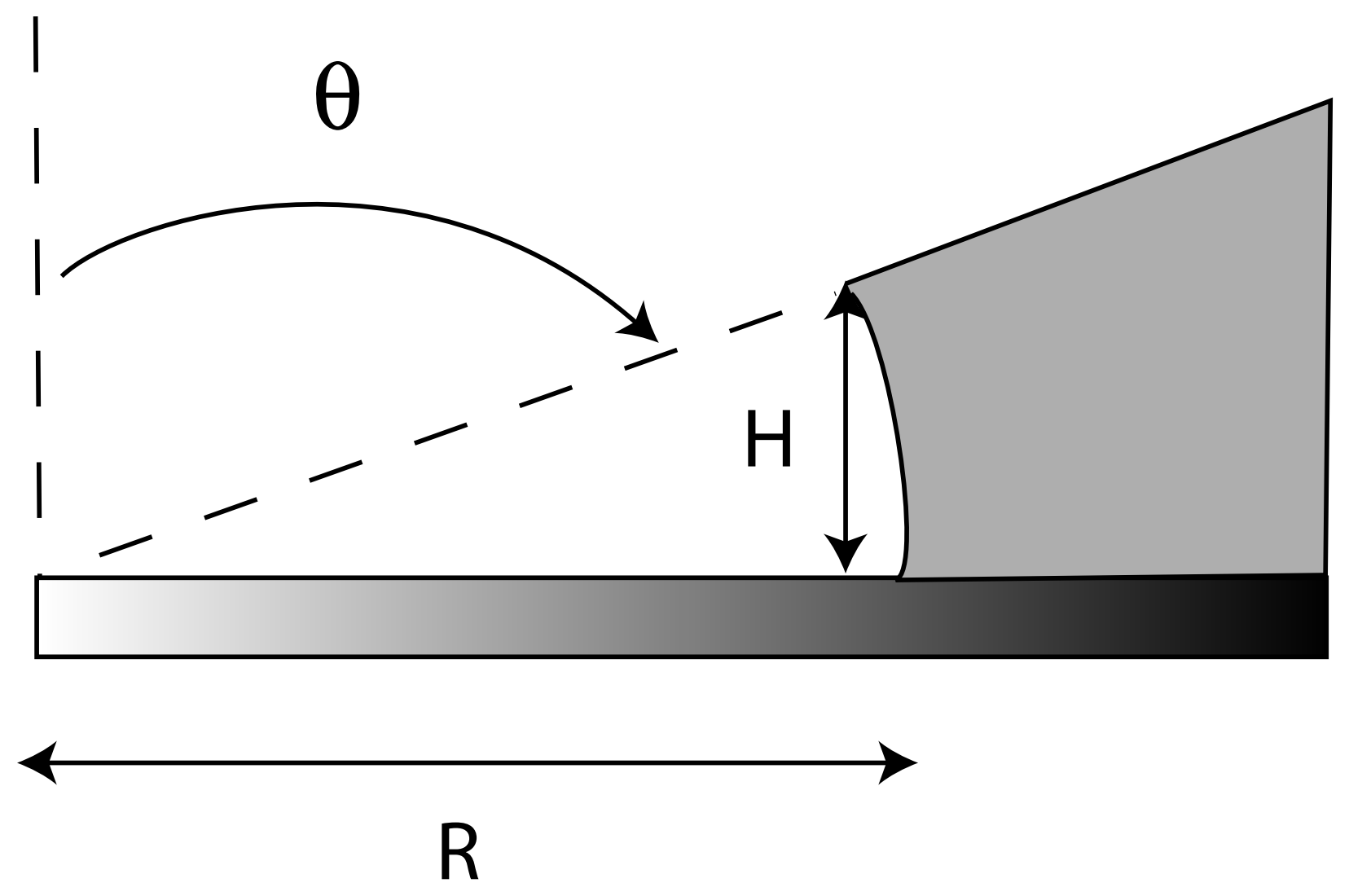

Fig. 6.- Geometry of the simple disk plus envelope model (see text). 


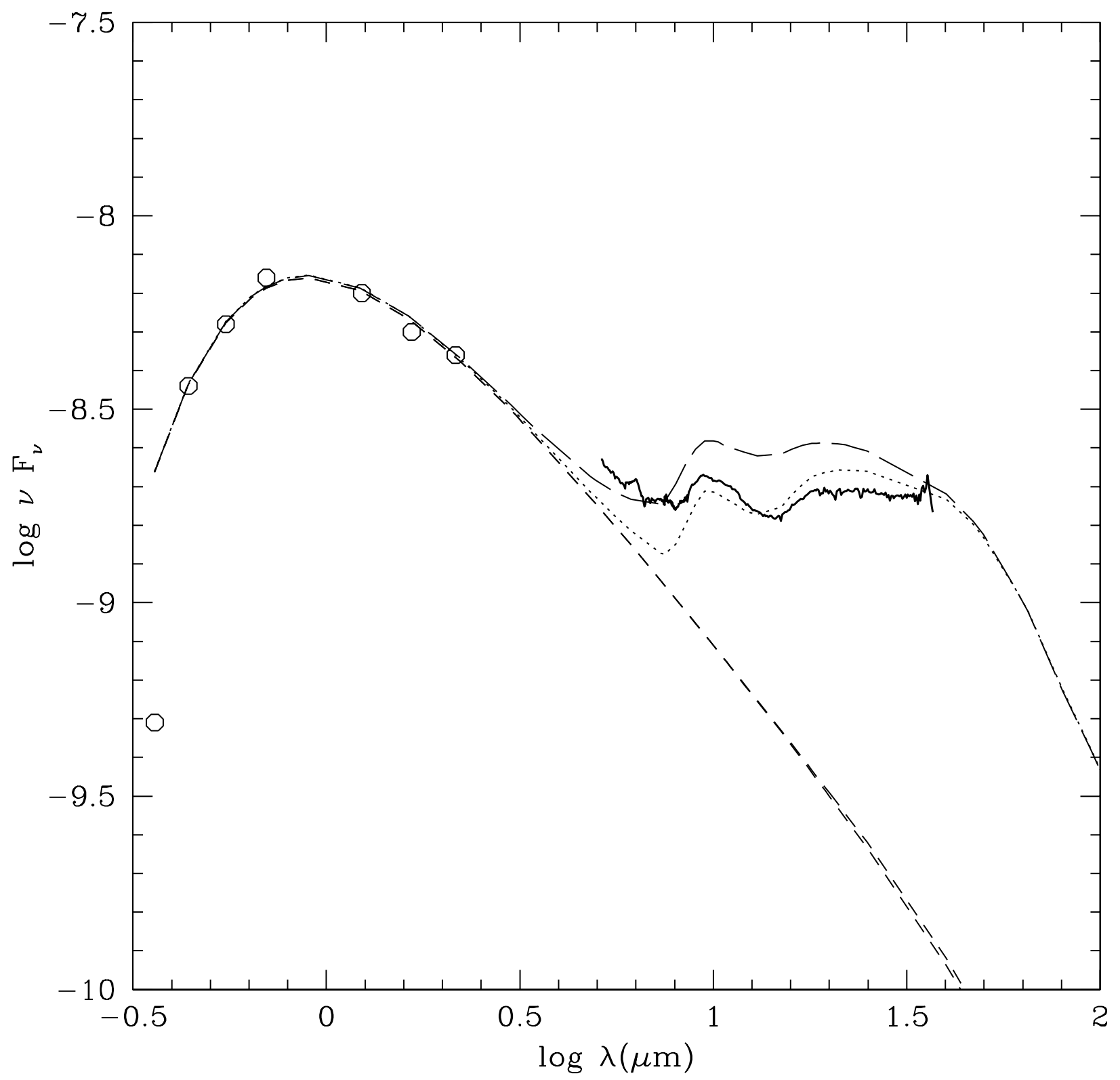

Fig. 7.- Envelope plus steady accretion disk models of V1057 Cyg from Hartmann et al. (1994) and Hartmann et al. (1996). The contribution of the disk alone (a series of annuli of blackbodies at a range of temperatures) is represented by the short dashed curve. The dotted curve shows the fluxes for a model with $\eta=3.0, \rho_{1}=10^{-13} \mathrm{gcm}^{-3}, R_{c}=20 \mathrm{AU}$, $r_{2}=7000 \mathrm{AU}, r_{1}=5 \mathrm{AU}$, and $z_{2}=4000 \mathrm{AU}$. This model fits the long-wavelength IRS range reasonably well but has too little flux shortward of $10 \mu \mathrm{m}$. The long-dashed curve is for a model in which the envelope is brought closer in $\left(r_{1}=3 \mathrm{AU}\right.$ and $R_{c}=10 \mathrm{AU}$; the other model parameters are identical to the previous case). This model is too bright in most of the IRS range, although it matches the short-wavelength end better. 


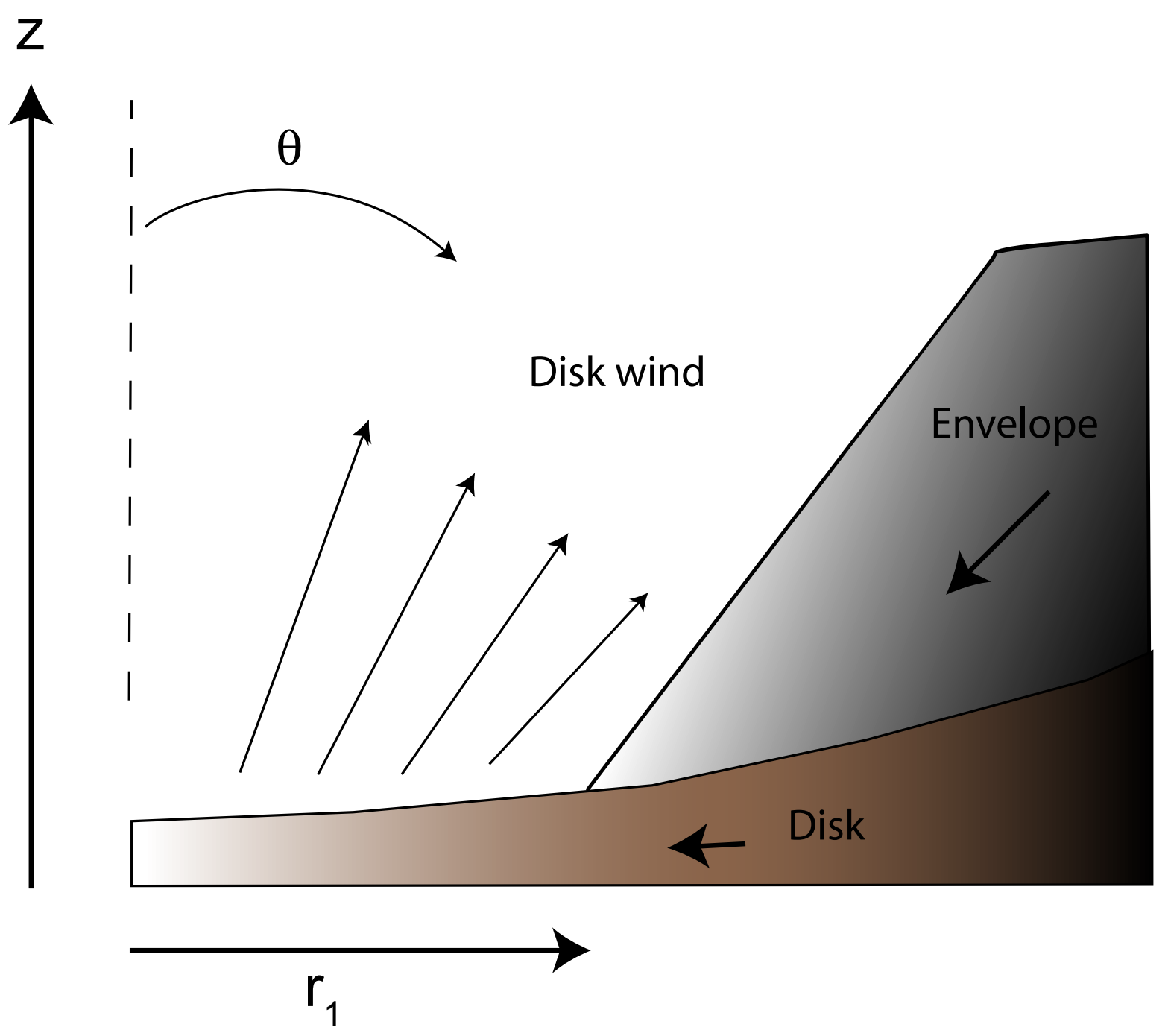

Fig. 8. - A schematic of the steady accretion disk plus envelope model from Figure 7. 
Table 1. Observations Times and AOR IDs

\begin{tabular}{lccc}
\hline \hline \multicolumn{1}{c}{ Object } & AORID & Date & JD \\
\hline V1057 Cyg & 3570176 & $2003-12-15$ & 2452988 \\
V346 Nor & 3570688 & $2004-02-27$ & 2453063 \\
FU Ori & 3569920 & $2004-03-04$ & 2453068 \\
BBW 76 & 3571200 & $2004-04-14$ & 2453110 \\
V1515 Cyg & 3570432 & $2004-05-11$ & 2453137 \\
\hline
\end{tabular}

Note. - Spitzer-IRS observation log of the FU Ori objects. 
Table 2. V1057 Cyg in 2004

\begin{tabular}{ccccc}
\hline \hline JD & V & U-B & B-V & V-R \\
\hline 53185.2727 & 12.361 & & 1.875 & 1.737 \\
53186.2465 & 12.368 & & 1.890 & 1.755 \\
53187.2281 & 12.364 & & 1.853 & 1.749 \\
53189.2199 & 12.374 & & 1.932 & 1.764 \\
53190.2565 & 12.388 & & 1.980 & 1.772 \\
53195.2785 & 12.444 & 1.484 & 1.892 & 1.769 \\
53196.2637 & 12.469 & 0.986 & 1.875 & 1.770 \\
53205.4167 & 12.463 & & 1.906 & 1.798 \\
53227.4443 & 12.402 & 1.804 & 1.958 & 1.759 \\
53228.3340 & 12.451 & 1.861 & 1.918 & 1.790 \\
53229.3367 & 12.450 & 2.266 & 1.942 & 1.789 \\
53230.2871 & 12.429 & 0.706 & 1.900 & 1.783 \\
53231.3084 & 12.441 & 1.858 & 1.873 & 1.795 \\
53232.2428 & 12.408 & & 1.939 & 1.759 \\
53233.2331 & 12.412 & 1.106 & 1.904 & 1.788 \\
53234.2302 & 12.366 & 1.665 & 1.976 & 1.767 \\
53235.3605 & 12.426 & & 1.939 & 1.736 \\
53236.3604 & 12.466 & 1.190 & 1.906 & 1.776 \\
53237.3542 & 12.495 & 1.418 & 1.876 & 1.806 \\
53238.2251 & 12.483 & 1.123 & 1.909 & 1.788 \\
53240.2332 & 12.474 & 1.123 & 1.907 & 1.779 \\
53241.3390 & 12.477 & 2.277 & 1.886 & 1.775 \\
53242.3228 & 12.499 & 1.616 & 1.956 & 1.793 \\
53243.3501 & 12.488 & & 1.935 & 1.773 \\
53250.2696 & 12.503 & & 1.937 & 1.821 \\
53251.2416 & 12.469 & 1.555 & 1.943 & 1.792 \\
53252.2603 & 12.480 & & 1.891 & 1.789 \\
53253.2421 & 12.458 & & 1.923 & 1.771 \\
53254.2550 & 12.487 & 1.032 & 1.933 & 1.794 \\
53255.2796 & 12.495 & 2.030 & 1.887 & 1.771 \\
53256.2439 & 12.491 & & 1.885 & 1.794 \\
53258.2582 & 12.480 & 1.590 & 1.928 & 1.767 \\
53259.3363 & 12.460 & & 1.912 & 1.774 \\
53260.2522 & 12.513 & 1.340 & 1.882 & 1.782 \\
53261.2235 & 12.485 & 1.958 & 1.905 & 1.767 \\
53262.2218 & 12.478 & 2.552 & 1.906 & 1.759 \\
53263.2217 & 12.446 & 1.384 & 1.952 & 1.756 \\
53263.2386 & 12.388 & & 2.096 & 1.747 \\
53264.2634 & 12.487 & 2.958 & 1.914 & 1.773 \\
53264.2891 & 12.527 & & 2.070 & 1.768 \\
53266.1891 & 12.513 & 1.854 & 2.016 & 1.766 \\
53266.2446 & 12.511 & 1.151 & 1.894 & 1.784 \\
53267.2124 & 12.482 & 1.375 & 1.909 & 1.771 \\
53267.2236 & 12.488 & 1.088 & 2.032 & 1.742 \\
53268.1896 & 12.537 & 1.237 & 1.975 & 1.785 \\
53270.2043 & 12.523 & & 1.904 & 1.763
\end{tabular}


Table 2-Continued

\begin{tabular}{ccccc}
\hline \hline JD & $\mathrm{V}$ & $\mathrm{U}-\mathrm{B}$ & $\mathrm{B}-\mathrm{V}$ & $\mathrm{V}-\mathrm{R}$ \\
\hline 53271.2405 & 12.537 & & 1.921 & 1.792 \\
53272.2170 & 12.486 & & 1.939 & 1.787 \\
53273.2196 & 12.490 & & 1.951 & 1.765 \\
53289.1960 & 12.600 & 2.405 & 2.013 & 1.802 \\
53291.2346 & 12.644 & & 1.955 & 1.835 \\
53292.2314 & 12.600 & 1.860 & 1.965 & 1.833 \\
53310.1500 & 12.771 & & 2.041 & 1.884 \\
\hline
\end{tabular}

Note. - The UBVR photometry for V1515 Cygni, FU Orionis and V1057 Cygni, was observed by Mansur Ibrahimov at Maidanak Observatory in 2004; the average magnitude is shown here. The UBVR photometry for BBW 76 is from Reipurth et al. (2002) and has been projected to 2004, using linear decline rates from the data (see text): U - $0.003 \mathrm{mag} y r^{-1}, \mathrm{~B}-0.014 \mathrm{mag} y r^{-1}$, $\mathrm{V}-0.023 \mathrm{mag} y r^{-1}, \mathrm{R}-0.026 \mathrm{mag} y r^{-1}$. Also included are estimated visual extinction correction factors for each object.

Table 3. FU Orionis in 2004

\begin{tabular}{ccccc}
\hline \hline JD & $\mathrm{V}$ & $\mathrm{U}-\mathrm{B}$ & $\mathrm{B}-\mathrm{V}$ & $\mathrm{V}-\mathrm{R}$ \\
\hline 53278.4601 & 9.654 & 0.682 & 1.361 & 1.169 \\
53280.4504 & 9.629 & 0.735 & 1.342 & 1.159 \\
53281.4718 & 9.639 & 0.799 & 1.349 & 1.173 \\
53283.4811 & 9.682 & 0.757 & 1.349 & 1.194 \\
53284.4826 & 9.677 & 0.748 & 1.347 & 1.173 \\
53299.4914 & 9.676 & 0.787 & 1.346 & 1.183 \\
53309.4760 & 9.659 & & 1.350 & 1.176 \\
53310.4598 & 9.661 & & 1.338 & 1.175 \\
53311.4993 & 9.652 & & 1.373 & 1.175 \\
\hline
\end{tabular}

Note. - Ground based data for FU Ori. 
Table 4. V1515 Cyg in 2004

\begin{tabular}{|c|c|c|c|c|}
\hline JD & V & U-B & B-V & V-R \\
\hline 53184.2758 & 12.973 & 0.620 & 1.599 & 1.509 \\
\hline 53185.2461 & 12.990 & & 1.597 & 1.531 \\
\hline 53186.2267 & 12.936 & & 1.563 & 1.485 \\
\hline 53187.2125 & 12.949 & & 1.566 & 1.477 \\
\hline 53189.2030 & 12.926 & & 1.764 & 1.502 \\
\hline 53190.2430 & 12.957 & & 1.741 & 1.536 \\
\hline 53193.2865 & 12.950 & 1.211 & 1.734 & 1.467 \\
\hline 53195.2580 & 12.866 & & & 1.483 \\
\hline 53196.2507 & 12.856 & 1.045 & 1.642 & 1.481 \\
\hline 53205.3837 & 13.048 & 1.056 & 1.634 & 1.523 \\
\hline 53224.3872 & 13.018 & 0.722 & 1.650 & 1.542 \\
\hline 53226.4111 & 13.062 & 1.302 & 1.640 & 1.530 \\
\hline 53227.3657 & 13.046 & 1.560 & 1.660 & 1.524 \\
\hline 53228.3002 & 13.052 & 1.228 & 1.688 & 1.518 \\
\hline 53229.2663 & 13.073 & 1.011 & 1.674 & 1.506 \\
\hline 53251.2222 & 13.054 & & 1.695 & 1.535 \\
\hline 53252.2431 & 13.115 & & 1.642 & 1.586 \\
\hline 53253.2262 & 13.116 & & 1.670 & 1.561 \\
\hline 53254.2282 & 13.118 & & 1.680 & 1.521 \\
\hline 53255.2192 & 13.175 & & 1.637 & 1.543 \\
\hline 53256.2161 & 13.160 & & 1.649 & 1.508 \\
\hline 53258.2446 & 13.199 & 1.185 & 1.665 & 1.562 \\
\hline 53259.3272 & 13.137 & 1.705 & 1.680 & 1.528 \\
\hline 53260.2360 & 13.121 & 2.027 & 1.697 & 1.543 \\
\hline 53261.2048 & 13.054 & 2.366 & 1.733 & 1.545 \\
\hline 53262.2066 & 13.066 & 1.968 & 1.645 & 1.545 \\
\hline 53263.2063 & 13.049 & 0.660 & 1.689 & 1.549 \\
\hline 53264.2492 & 13.064 & 1.191 & 1.683 & 1.520 \\
\hline 53266.2246 & 13.071 & 1.865 & 1.636 & 1.564 \\
\hline 53267.1977 & 13.033 & 1.379 & 1.660 & 1.505 \\
\hline 53268.2515 & 12.977 & & 1.664 & 1.489 \\
\hline 53270.1930 & 12.952 & & 1.624 & 1.507 \\
\hline 53271.2320 & 12.963 & & 1.643 & 1.490 \\
\hline 53272.1869 & 13.011 & & 1.586 & 1.527 \\
\hline 53273.1885 & 13.039 & & 1.654 & 1.530 \\
\hline 53289.1777 & 13.108 & 1.347 & 1.632 & 1.536 \\
\hline 53292.1552 & 13.042 & 0.912 & 1.615 & 1.489 \\
\hline
\end{tabular}

Note. - Ground-based data for V1515 Cyg. 
Table 5. Estimated 2004 Brightness Levels and Visual Extinction

\begin{tabular}{lrrrrr}
\hline \hline Object & $\mathrm{U}(\mathrm{mag})$ & $\mathrm{B}(\mathrm{mag})$ & $\mathrm{V}(\mathrm{mag})$ & $\mathrm{R}(\mathrm{mag})$ & $A_{V}$ \\
\hline V1057 Cyg & 17.00 & 14.70 & 12.65 & 10.85 & 3.7 \\
FU Ori & 11.81 & 11.01 & 9.67 & 8.49 & 1.9 \\
BBW 76 & 14.23 & 14.04 & 12.59 & 11.81 & 2.2 \\
V1515 Cyg & 16.50 & 14.74 & 13.11 & 11.57 & 3.2 \\
\hline
\end{tabular}

Note. - Current UBVR photometry and uncorrected for reddening, and assumed reddening corrections for the FU Ori objects. The average values for V1057 Cyg, V1515 Cyg, and FU Ori are computed from tables 2, 3, and 4. The values for BBW 76 are extrapolations from older data, as explained in the text.

Table 6. Steady Accretion Model Parameters

\begin{tabular}{lrrrrr}
\hline \hline Object & $\mathrm{d}(\mathrm{kpc})$ & $L / L_{\odot}$ & $T(\max )$ & $R_{i} / R_{\odot}$ & $M \dot{M} /\left(10^{-4} M_{\odot}^{2} \mathrm{yr}-1\right)$ \\
\hline FU Ori & 0.5 & 466 & 7710 & 5.0 & 1.5 \\
V1057 Cyg & 0.6 & 170 & 6590 & 3.7 & 0.45 \\
V1515 Cyg & 1.0 & 177 & 7710 & 3.1 & 0.35 \\
BBW 76 & 1.8 & 287 & 7710 & 3.9 & 0.72 \\
\hline
\end{tabular}

Note. - Distances are from Hartmann \& Kenyon (1996), except for BBW 76, which is taken from Reipurth et al. (2002). Note: if V1057 Cyg and V1515 Cyg are observed pole-on, then the true accretion luminosities may be a factor of up to two smaller, with a reduction of $2^{1 / 2}$ in the inner radius and a reduction of $2^{3 / 2}$ in $M \dot{M}$. 